Acta Crystallographica Section E

Structure Reports

Online

ISSN 1600-5368

\section{Susan J. Berners-Price, $\neq$ \\ Richard J. Bowen§ and Peter C. Healy*}

School of Science, Griffith University, Nathan, Brisbane 4111, Australia

\# Present address: School of Biomedical and Chemical Sciences, The University of Western Australia, Crawley, Perth 6009, Australia. $\S$ Present address: Mintek, Private Bag X3015, Randburg 2125, South Africa.

Correspondence e-mail: p.healy@griffith.edu.au

\section{Key indicators}

Single-crystal X-ray study

$T=295 \mathrm{~K}$

Mean $\sigma(\mathrm{C}-\mathrm{C})=0.006 \AA$

$R$ factor $=0.052$

$w R$ factor $=0.182$

Data-to-parameter ratio $=18.1$

For details of how these key indicators were automatically derived from the article, see http://journals.iucr.org/e.

\title{
1,2-Bis(di-4-pyridylphosphino)ethane (d4pype)
}

The title compound (d4pype), $\mathrm{C}_{22} \mathrm{H}_{20} \mathrm{~N}_{4} \mathrm{P}_{2}$, crystallizes as a discrete molecular species disposed about a crystallographic inversion centre at the mid-point of the central $\mathrm{C}-\mathrm{C}$ bond.

\section{Comment}

Bidentate tertiary phosphine ligands with pyridyl substituents, such as the title compound, (I), are of interest because a number of studies have shown that metal complexes with these ligands exhibit selective anti-tumour properties (Berners-Price et al., 1999; McKeage et al., 2000). During the course of our work in this area, we obtained crystals of (I) which were suitable for X-ray diffraction studies.

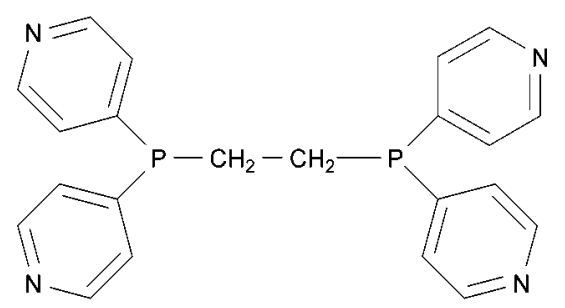

(I)

Compound (I) crystallizes in space group $P 2_{1} / n$ as discrete molecules disposed in a trans configuration about a crystallographic inversion centre at the mid-point of the central C$\mathrm{C}$ bond (Fig. 1). Noteworthy features of the geometric parameters in the structure are the $\mathrm{P}-\mathrm{C}(\mathrm{py})$ bond lengths of 1.842 (3) and 1.837 (4) $\AA$, which are similar to values of 1.846 (3) and 1.850 (3) $\AA$ for the structure of 1,2-bis(di-2pyridylphosphino)ethane (Jones et al., 1999) but longer than bond lengths of 1.818 (4) and 1.829 (3) A observed for the P$\mathrm{C}(\mathrm{Ph})$ bonds in 1,2-bis(diphenylphosphino)ethane (Pelizzi \& Pelizzi, 1979). The $\mathrm{C}-\mathrm{N}$ bond lengths in the pyridyl rings range between 1.317 (5) and 1.337 (5) $\AA$, which are characteristic for analogous bond lengths in other pyridine and pyridyl systems (e.g. Brammer \& Zhao, 1995; Jones et al., 1999). All other bond lengths and angles are in accord with expected values.

\section{Experimental}

1,2-Bis(di-4-pyridylphosphino)ethane was prepared according to published procedures (Bowen et al., 1998). Single crystals suitable for $\mathrm{X}$-ray crystallographic analysis were obtained as a by-product of slow evaporation of a solution of copper(I) chloride and (I) (molar ratio 1:2) in an acetonitrile/dichloromethane mixture. 


\section{Crystal data}

$$
\begin{aligned}
& \mathrm{C}_{22} \mathrm{H}_{20} \mathrm{~N}_{4} \mathrm{P}_{2} \\
& M_{r}=402.37 \\
& \text { Monoclinic, } P 2_{1} / n \\
& a=14.073(6) \AA \\
& b=8.228(2) \AA \\
& c=9.200(2) \AA \\
& \beta=108.33(3)^{\circ} \\
& V=1011.2(6) \AA^{3} \\
& Z=2
\end{aligned}
$$

Data collection

$\begin{array}{ll}\text { Rigaku AFC- } 7 R \text { diffractometer } & \theta_{\max }=27.5^{\circ} \\ \omega / 2 \theta \text { scans } & h=-7 \rightarrow 18 \\ \text { Absorption correction: none } & k=0 \rightarrow 10 \\ 2552 \text { measured reflections } & l=-11 \rightarrow 11 \\ 2323 \text { independent reflections } & 3 \text { standard reflections } \\ 1170 \text { reflections with } I>2 \sigma(I) & \text { every } 150 \text { reflections } \\ R_{\text {int }}=0.038 & \text { intensity decay: } 0.9 \%\end{array}$

\section{Refinement}

Refinement on $F^{2}$

$R\left[F^{2}>2 \sigma\left(F^{2}\right)\right]=0.052$

$w R\left(F^{2}\right)=0.182$

$S=1.00$

2323 reflections

128 parameters

\section{Table 1}

Selected geometric parameters $\left(\AA,{ }^{\circ}\right)$.

\begin{tabular}{llll}
\hline $\mathrm{P} 1-\mathrm{C} 1$ & $1.842(3)$ & $\mathrm{C} 1-\mathrm{C} 5$ & $1.381(5)$ \\
$\mathrm{P} 1-\mathrm{C} 6$ & $1.837(4)$ & $\mathrm{C} 2-\mathrm{C} 3$ & $1.385(6)$ \\
$\mathrm{P} 1-\mathrm{C} 11$ & $1.849(4)$ & $\mathrm{C} 4-\mathrm{C} 5$ & $1.385(6)$ \\
$\mathrm{N} 1-\mathrm{C} 3$ & $1.337(5)$ & $\mathrm{C} 6-\mathrm{C} 7$ & $1.387(6)$ \\
$\mathrm{N} 1-\mathrm{C} 4$ & $1.328(5)$ & $\mathrm{C} 6-\mathrm{C} 10$ & $1.367(6)$ \\
$\mathrm{N} 2-\mathrm{C} 8$ & $1.317(7)$ & $\mathrm{C} 7-\mathrm{C} 8$ & $1.383(7)$ \\
$\mathrm{N} 2-\mathrm{C} 9$ & $1.318(7)$ & $\mathrm{C} 9-\mathrm{C} 10$ & $1.386(7)$ \\
$\mathrm{C} 1-\mathrm{C} 2$ & $1.388(5)$ & $\mathrm{C} 11-\mathrm{C} 11^{\mathrm{i}}$ & $1.526(6)$ \\
& & & \\
$\mathrm{C} 1-\mathrm{P} 1-\mathrm{C} 6$ & $100.04(15)$ & $\mathrm{N} 1-\mathrm{C} 4-\mathrm{C} 5$ & $124.1(4)$ \\
$\mathrm{C} 1-\mathrm{P} 1-\mathrm{C} 11$ & $102.74(16)$ & $\mathrm{C} 1-\mathrm{C} 5-\mathrm{C} 4$ & $120.0(4)$ \\
$\mathrm{C} 6-\mathrm{P} 1-\mathrm{C} 11$ & $102.61(18)$ & $\mathrm{P} 1-\mathrm{C} 6-\mathrm{C} 7$ & $117.4(3)$ \\
$\mathrm{C} 3-\mathrm{N} 1-\mathrm{C} 4$ & $115.9(4)$ & $\mathrm{P} 1-\mathrm{C} 6-\mathrm{C} 10$ & $126.0(3)$ \\
$\mathrm{C} 8-\mathrm{N} 2-\mathrm{C} 9$ & $114.9(5)$ & $\mathrm{C} 7-\mathrm{C} 6-\mathrm{C} 10$ & $116.5(4)$ \\
$\mathrm{P} 1-\mathrm{C} 1-\mathrm{C} 2$ & $118.3(3)$ & $\mathrm{C} 6-\mathrm{C} 7-\mathrm{C} 8$ & $119.1(4)$ \\
$\mathrm{P} 1-\mathrm{C} 1-\mathrm{C} 5$ & $125.3(3)$ & $\mathrm{N} 2-\mathrm{C} 8-\mathrm{C} 7$ & $125.0(5)$ \\
$\mathrm{C} 2-\mathrm{C} 1-\mathrm{C} 5$ & $116.3(3)$ & $\mathrm{N} 2-\mathrm{C} 9-\mathrm{C} 10$ & $125.0(5)$ \\
$\mathrm{C} 1-\mathrm{C} 2-\mathrm{C} 3$ & $119.8(4)$ & $\mathrm{C} 6-\mathrm{C} 10-\mathrm{C} 9$ & $119.4(4)$ \\
$\mathrm{N} 1-\mathrm{C} 3-\mathrm{C} 2$ & $123.8(4)$ & $\mathrm{P} 1-\mathrm{C} 11-\mathrm{C} 11^{\mathrm{i}}$ & $112.4(3)$ \\
\hline
\end{tabular}

Symmetry code: (i) $2-x, 1-y, 2-z$.

$\mathrm{H}$ atoms were constrained in the riding model approximation, fixed to their parent $\mathrm{C}$ atoms at a $\mathrm{C}-\mathrm{H}$ distance of $0.95 \AA$, and $U_{\text {iso }}(\mathrm{H})$ values were set to $1.2 U_{\text {eq }}$ of the parent atom.

Data collection: MSC/AFC-7 Diffractometer Control Software for Windows (Molecular Structure Corporation, 1999); cell refinement: MSC/AFC-7 Diffractometer Control Software for Windows; data

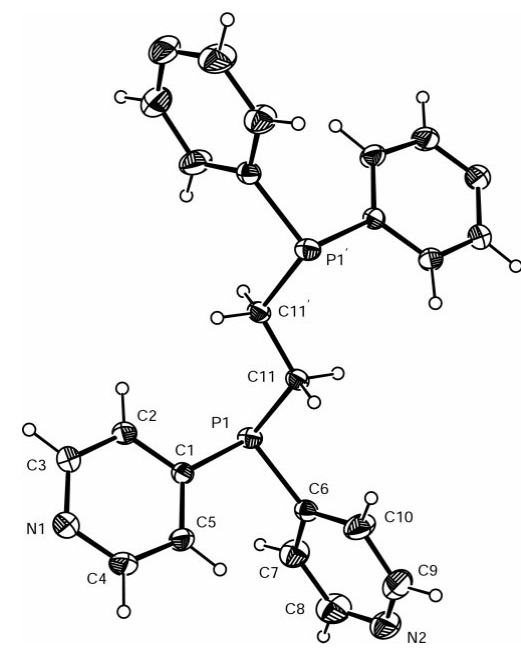

Figure 1

View of the title compound, with the atom-numbering scheme. Displacement ellipsoids for non-H atoms are drawn at the $30 \%$ probability level.

reduction: TEXSAN for Windows (Molecular Structure Corporation, 1997-2001); program(s) used to solve structure: TEXSAN for Windows; program(s) used to refine structure: TEXSAN for Windows and SHELXL97 (Sheldrick, 1997); molecular graphics: ORTEP-3 (Farrugia, 1997); software used to prepare material for publication: TEXSAN for Windows and PLATON (Spek, 1980-2001).

The authors thank Griffith University for financial assistance.

\section{References}

Berners-Price, S. J., Bowen, R. J., Galettis, P., Healy, P. C. \& McKeage, M. J. (1999). Coord. Chem. Rev. 185-186, 823-836.

Bowen, R. J., Garner, C., Berners-Price, S. J., Jenkins, I. D. \& Sue, R. E. (1998). J. Organomet. Chem. 554, 181-184.

Brammer, L. \& Zhao, D. (1995). Acta Cryst. C51, 45-48.

Farrugia, L. J. (1997). J. Appl. Cryst. 30, 565.

Jones, N. D., MacFarlane, K. S., Smith, M. B., Schutte, R. P., Rettig, S. J. \& James, B. R. (1999). Inorg. Chem. 38, 3956-3966.

McKeage, M. J., Berners-Price, S. J., Galettis, P., Bowen, R. J., Brouwer, W., Ding, L., Baguley, B. C. (2000). Cancer Chemother. Pharmocol. 46, 343-350. Molecular Structure Corporation (1999). MSC/AFC-7 Diffractometer Control Software for Windows. Version 1.02. MSC, 9009 New Trails Drive, The Woodlands, TX 77381, USA.

Molecular Structure Corporation (1997-2001). TEXSAN for Windows. Version 1.06. MSC, 9009 New Trails Drive, The Woodlands, TX 77381, USA. Pelizzi, C. \& Pelizzi, G. (1979). Acta Cryst. B35, 1785-1790.

Sheldrick, G. M. (1997). SHELXL97. University of Göttingen, Germany. Spek, A. L. (1980-2001). PLATON for Windows. Version 121201. University of Utrecht, The Netherlands. 